\title{
Medical Evaluation of Children with Intellectual Disability: Clinician Compliance with Published Guidelines
}

\author{
Alexis Tchaconas ${ }^{1}$ and Andrew Adesman ${ }^{1,2, *}$ \\ ${ }^{1}$ Pediatrics, Cohen Children's Medical Center of New York, New Hyde Park, 1983 Marcus Avenue, Suite 130, \\ Lake Success, NY 11042, USA \\ ${ }^{2}$ Pediatrics, Hofstra North Shore-LIJ School of Medicine, Hempstead, 500 Hofstra University, Hempstead, NY \\ 11549, USA
}

\begin{abstract}
Background: Children with intellectual disability (CWID) in the USA are typically referred to child neurologists (CN) and developmental-behavioral pediatricians (DBP) for medical evaluation. Although the American Academy of Neurology/Child Neurology Society (AAN/CNS) and the American Academy of Pediatrics (AAP) have published evaluation guidelines, experience suggests $\mathrm{CN}$ and DBP do not consistently follow them. Our goal was to assess CN's and DBP's approach to evaluating CWID and overall compliance with published guidelines.
\end{abstract}

Methods: Questionnaires were mailed to $C N$ and DBP in the U.S. $(n=1897)$. Physicians were asked demographic information and which laboratory tests they would "routinely order" for the hypothetical case of a $3 \frac{1}{2}$ year old boy with Full Scale IQ=58 and unremarkable neurological history and exam. Chi-square tests were performed to compare sub-specialists' ordering practices.

Results: $127 \mathrm{CN}$ and 140 DBP responded. $7.1 \% \mathrm{CN}(\mathrm{n}=9)$ and $11.4 \% \mathrm{DBP}(\mathrm{n}=16)$ complied with AAN/CNS and AAP guidelines, respectively. Although routinely indicated, $36.2 \% \mathrm{CN}$ and $31.4 \% \mathrm{DBP}$ would not routinely order chromosomal microarray (CMA), and $42.5 \% \mathrm{CN}$ and $26.4 \%$ DBP would not routinely order DNA for Fragile $X\left(X^{2}=7.67, p=0.006\right) .7 .9 \%$ $\mathrm{CN}$ and $7.1 \%$ DBP would order a karyotype without CMA. Although not indicated, $7.1 \% \mathrm{CN}$ and $0.7 \%$ DBP noted they would routinely order an EEG $\left(x^{2}=7.50, p=0.006\right)$. A brain MRI is only recommended by AAN/CNS guidelines; $49.6 \% \mathrm{CN}$ and $12.9 \%$ DBP reported they would routinely order it $\left(X^{2}=42.55, p<.0001\right)$.

Conclusion: Few CN and DBP follow published guidelines for laboratory evaluation of CWID. Relative to DBP, CN more frequently order EEGs and MRIs but less frequently order recommended genetic tests.

Keywords: Intellectual disability, etiology, clinical practice guidelines, medical evaluation, chromosomal microarray.

\section{INTRODUCTION}

Intellectual disability (ID) is a developmental disorder marked by an intelligence quotient (IQ) of less than around 70 , significant deficits in cognitive functioning, and limitations in adaptive skills in two or more of the following developmental domains: social skills, community living, communication, home living, health, self-direction, work, and leisure [1-4]. The DSM5 recently redefined ID as impairments of general mental capabilities that affect adaptive functioning in three domains: conceptual (i.e. language, reading, memory), social (i.e. empathy, interpersonal communication, social judgment), and practical (i.e. personal care, money management, organization of tasks) [5]. In infants and young children, ID is hard to diagnose given the lack of valid and reliable IQ scores and the difficulty in assessing adaptive function $[6,7]$. Accordingly, children less than 6 years old that are believed to be at risk for ID are diagnosed with global developmental delay (GDD) [8]. For the purposes of

*Address correspondence to this author at the Pediatrics, Hofstra North ShoreLIJ School of Medicine, Hempstead, 500 Hofstra University, Hempstead, NY 11549, USA; E-mail: aadesman@nshs.edu this study, ID and GDD are considered interchangeable, given that the etiological medical evaluation of both disorders is identical [7-10].

ID affects between $1-3 \%$ of the US population with symptoms that begin during the developmental period before 18 years of age $[5,9,11,12]$. Although a primary care pediatrician may often identify GDD or ID in a young child based on clinical assessment and review of formal developmental testing [13], evaluation of children with ID (CWID) for a medical etiology is generally pursued by pediatric sub-specialists, such as child neurologists, developmental pediatricians, and clinical geneticists. The etiological evaluation of ID generally consists of genetic testing for chromosomal abnormalities or mutations in genes known to affect intellectual development and, if indicated, metabolic testing for inborn errors of metabolism, which can include biological errors in amino acid, carbohydrate, purine, peptide, and mitochondrial metabolism [7-9]. In more complex cases with frank neuropathology such as seizures and/or developmental regression, neuroimaging tests and electroencephalograms (EEG) should also be performed [7]. The purpose of identifying the underlying etiology of ID is not only to alleviate family stress, but also to provide an early and 
targeted management plan with viable treatment options (if available), in addition to informed counseling about prognosis and recurrence risk [14].

Efforts have been made to standardize the etiological laboratory evaluation of ID among pediatric specialists. To facilitate this standardization, medical specialty societies have periodically released their own clinical guidelines for evaluating and managing patients with specific disorders. Specifically, in 2014, the American Academy of Pediatrics (AAP) published guidelines [7] as an update to earlier clinical recommendations made in 2006 [15, 16]. The American Academy of Neurology/Child Neurology Society's (AAN/CNS) current guidelines are over a decade old [8]; however, the AAN/CNS have published more recent updates on genetic testing for the etiological evaluation of ID in 2011 [9] and 2013 [17].

Table 1: Recommended Laboratory Tests for the Routine Etiological Evaluation of a Child with Non-Complicated, Otherwise Unexplained Intellectual Disability



${ }^{\text {a }}$ The AAP recommends consideration of specific metabolic testing after a CMA is performed, which should include serum total homocysteine, acyl-carnitine profile, amino acids; and urine organic acids, glycosaminoglycans, oligosaccharides, purines, pyrimidines, GAA/creatine metabolites (Moeschler \& Shevell, 2014).

${ }^{b}$ If universal newborn screening was not previously performed, clinicians are told to consider the following metabolic tests before proceeding with the stepwise evaluation: urine organic acid screen, quantitative serum amino acids, serum lactate and ammonia levels, capillary or arterial blood gas, and thyroid function studies (Shevell et al., 2003). These studies can also be used for later metabolic testing. 
The AAP guidelines recommend the following evaluative approach: comprehensive medical evaluation and physical examination with dysmorphologic and neurologic examinations, genetic testing (CMA and DNA for Fragile $X)$ and consideration of case-specific metabolic testing (see Table 1) [7]. If no diagnosis is established from these tests, the AAP recommends a complete X-linkage Intellectual Disability (XLID) panel for males with a family history suggestive of an X-linkage disorder, and a complete MECP2 deletion, duplication, and sequencing study (testing for Rett syndrome) for females [7]. An MRI is only advised if there are abnormal findings on neurologic exam (e.g., focal findings), microcephaly, or macrocephaly [7].

While the 2003 AAN/CNS guidelines also recommend an initial comprehensive history and physical examination, the guidelines largely depart from those released by the AAP. Table 1 provides a thorough comparison of the AAP and AAN/CNS clinical guidelines for evaluating the etiology of idiopathic cases of ID, as well as recommended tests to order in the presence of additional clinical symptoms.

The 2003 AAN/CNS guidelines [8] recommend that child neurologists conduct a stepwise evaluation consisting of a brain MRI, karyotype, genetic testing for Fragile $X$, metabolic testing, genetic testing for subtelomeric rearrangements, and, for females, genetic testing for Rett Syndrome. However, the AAN/CNS also published an evidence report in 2011 [9], which recommends the CMA as a first-line genetic test (instead of a karyotype).

Although guidelines for the medical evaluation of ID exist, it is uncertain to what extent pediatric specialists actually comply with these guidelines. While previous studies have investigated clinicians' compliance with clinical practice guidelines for other conditions such as non-febrile seizures [18] and depression [19, 20], there has never been a systematic study examining child neurologists' and developmental-behavioral pediatricians' approach to the laboratory evaluation of children with ID and assessing their compliance with extant guidelines.

The purpose of our study was to identify what tests $\mathrm{CN}$ and DBP routinely order when evaluating CWID, to assess to what extent they comply with clinical practice guidelines, and to identify any differences between these two sub-specialists in their evaluation practices.

\section{METHODS}

\section{Study Design}

A 2-page questionnaire was mailed to a national sample of $\mathrm{CN}$ and DBP. The first section contained demographic questions: physician's gender, boardcertification status, number of years practicing in their sub-specialty, geographic location of the practice, and type of setting where practice is based. The second section of the questionnaire presented 3 hypothetical cases of children with developmental disorders and asked which laboratory tests the physician would order based on each child's medical history and clinical features. This paper reports results for the case of a $31 / 2$ year old boy with Full Scale $I Q=58$, no history of seizures, vomiting, or lethargy, no dysmorphic features, and head circumference at the $90^{\text {th }}$ percentile (with height at the $50^{\text {th }}$ percentile). The laboratory tests were grouped into three categories: EEG \& neuroimaging, genetic tests, and other tests (metabolic). The tests included in the questionnaire were a mix of tests recommended by the AAN/CNS and/or AAP guidelines or commonly ordered though not endorsed by guidelines. The third section of the questionnaire asked the physicians how often they refer children with moderately severe autism or ID to various other subspecialists for diagnostic testing.

\section{Subjects}

Questionnaires were mailed to all DBP in the Society for Developmental \& Behavioral Pediatrics $(n=700)$ and to all $C N$ in clinical practice $(n=1197)$ from the American Medical Association's master list of physicians in the United States.

The mailing to the pediatric sub-specialists included a cover letter explaining the study, the questionnaire, and a prepaid return envelope. As compensation for their time, physicians were also given a copy of the ADHD Medication Guide, which is a patient education visual aid that they were allowed to keep regardless of their decision to participate. Study participation was voluntary and completely anonymous. This study was approved by the Institutional Review Board of the North Shore-LIJ Health System.

\section{Compliance with Guidelines}

CN's etiologic testing practices were evaluated with respect to the AAN/CNS's 2003 guidelines and also with respect to the 2011 evidence report, while DBP's 
testing practices were assessed relative to the AAP's 2014 guidelines.

\section{Statistical Methods}

The percentage of physicians in each sub-specialty who would "routinely order" each of the tests was calculated. Chi-square analysis or Fisher's Exact test were applied to the data to determine if a significant difference exists between $\mathrm{CN}$ and $\mathrm{DBP}$ in their demographics and adherence to guidelines for ID evaluation. Results with $p<0.05$ were considered significant.

\section{RESULTS}

Responses were received from 140 CN (11.7\% response rate) and $181 \mathrm{DBP}$ (25.9\% response rate), yielding a $16.9 \%$ overall response rate. Of the 321 respondents, 267 (127 CN and $140 \mathrm{DBP})$ met the criteria for inclusion in our analysis: currently practices in the United States and is board-certified in child neurology and/or developmental pediatrics (includes developmental and behavioral pediatrics and neurodevelopmental disabilities). Our final sample for analysis represents $14.1 \%$ of our original mailing. Demographic characteristics for the sample are listed in Table 2. When comparing $\mathrm{CN}$ and $\mathrm{DBP}$, no differences were noted in practice duration or setting. The DBP respondents were predominantly female, whereas the $\mathrm{CN}$ were predominantly male $\left(x^{2}=11.49\right.$, $\mathrm{p}=0.0007)$.

Tables 3 and 4 list the percentage of $\mathrm{CN}$ and DBP that would routinely order each test to etiologically evaluate the hypothetical case of a $3 \frac{1}{2}$ year old boy with mild ID. Table 3 contains the tests recommended by at least one set of guidelines, while Table $\mathbf{4}$ contains the tests not recommended by either set of guidelines.

\section{Genetic Testing}

The majority of respondents indicated they would order one or more recommended genetic tests. Specifically, two-thirds stated they would order DNA for Fragile $X(65.5 \%)$ and a similar percentage would order a CMA (66.3\%). 55.4\% indicated they would do both a CMA and DNA for Fragile X. Conversely, 23.2\% of the entire sample stated they would do neither test, and $22.1 \%$ reported they would not do any genetic testing whatsoever $\left(25.2 \% \mathrm{CN}\right.$ vs. $\left.19.3 \% \mathrm{DBP} ; \mathrm{x}^{2}=1.35, \mathrm{~ns}\right)$.

When comparing genetic testing for the two subspecialties, DBP were significantly more likely than $\mathrm{CN}$

Table 2: Sub-Specialist Demographic and Practice Information ( $N=267)$

\begin{tabular}{|c|c|c|c|c|c|c|c|c|c|c|}
\hline & \multicolumn{2}{|c|}{$\begin{array}{c}C N \\
(N=127)\end{array}$} & \multicolumn{2}{|c|}{$\begin{array}{c}\text { DBP } \\
(\mathrm{N}=140)\end{array}$} & \multicolumn{2}{|c|}{ DBP vs. CN } & \multicolumn{2}{|c|}{$\begin{array}{c}\text { Total } \\
(\mathrm{N}=\mathbf{2 6 7})\end{array}$} & \multicolumn{2}{|c|}{ Overall } \\
\hline & $\mathbf{N}$ & $\%$ & $\mathbf{N}$ & $\%$ & $x^{2}$ & p-value & $\mathbf{N}$ & $\%$ & $x^{2}$ & $p$-value \\
\hline Gender $^{a}$ & & & & & & & & & 2.16 & n.s. \\
\hline Males & 79 & 66.4 & 63 & 45.3 & 11.49 & 0.0007 & 142 & 53.2 & & \\
\hline $\begin{array}{l}\text { Years in } \\
\text { Practice }^{b}\end{array}$ & & & & & & & & & 71.58 & $<0.0001$ \\
\hline$<5$ & 27 & 21.3 & 20 & 14.9 & 1.77 & n.s. & 47 & 18.0 & & \\
\hline $5-15$ & 35 & 27.6 & 33 & 24.6 & 0.29 & n.s. & 68 & 26.1 & & \\
\hline $16-30$ & 46 & 36.2 & 66 & 49.3 & 4.52 & 0.03 & 112 & 42.9 & & \\
\hline$>30$ & 19 & 14.9 & 15 & 11.2 & 0.82 & n.s. & 34 & 13.0 & & \\
\hline Practice Type $^{c}$ & & & & & & & & & 311.5 & $<0.0001$ \\
\hline Private office & 42 & 33.3 & 32 & 22.4 & 4.03 & 0.04 & 74 & 27.5 & & \\
\hline $\begin{array}{l}\text { Community } \\
\text { clinic }\end{array}$ & 5 & 4.0 & 15 & 10.5 & 4.14 & 0.04 & 20 & 7.4 & & \\
\hline Hospital-based & 70 & 55.5 & 77 & 53.8 & 0.08 & n.s. & 147 & 54.7 & & \\
\hline School & 3 & 2.4 & 12 & 8.4 & 4.60 & 0.03 & 15 & 5.6 & & \\
\hline Other & 6 & 4.8 & 7 & 4.9 & 0.003 & n.s. & 13 & 4.8 & & \\
\hline
\end{tabular}

${ }^{\mathrm{a}} \mathbf{N}=139 \mathrm{DBP}$ and $119 \mathrm{CN}$

${ }^{\mathbf{b}} \mathbf{N}=134 \mathrm{DBP}$ and $127 \mathrm{CN}$.

${ }^{\mathrm{C}} \mathrm{N}=143 \mathrm{DBP}$ and $126 \mathrm{CN}$; Multiple responses allowed.

n.s. = non-significant $p$-value. 
Table 3: Percent of Sub-Specialists Ordering Recommended Laboratory Tests for the Routine Etiologic Evaluation of Intellectual Disability

\begin{tabular}{|c|c|c|c|}
\hline \multicolumn{4}{|l|}{ Genetics } \\
\hline DNA for Fragile $\mathrm{X}^{\mathrm{a}, \mathrm{b}, \mathrm{d}}$ & $57.5 \%(\mathrm{~N}=73)$ & $73.6 \%(\mathrm{~N}=103)$ & $7.67, p=0.006$ \\
\hline Chromosomal microarray ${ }^{\mathrm{b}, \mathrm{d}}$ & $63.8 \%(\mathrm{~N}=81)$ & $68.6 \%(\mathrm{~N}=96)$ & 0.68, n.s. \\
\hline - Chromosomal microarray and karyotype & $24.4 \%(\mathrm{~N}=31)$ & $35.7 \%(N=50)$ & $4.03, p=0.04$ \\
\hline - Chromosomal microarray and not karyotype & $39.4 \%(N=50)$ & $32.9 \%(\mathrm{~N}=46)$ & 1.23, n.s. \\
\hline \multicolumn{4}{|l|}{ Neuroimaging } \\
\hline Brain $M R I^{a}$ & $49.6 \%(\mathrm{~N}=63)$ & $12.9 \%(\mathrm{~N}=18)$ & $42.55, p<0.0001$ \\
\hline \multicolumn{4}{|l|}{ Metabolic } \\
\hline Urine Organic Acids ${ }^{c, e}$ & $27.6 \%(\mathrm{~N}=35)$ & $18.6 \%(\mathrm{~N}=26)$ & $3.05, p=0.08$ \\
\hline Serum Amino Acids ${ }^{\mathrm{c}, \mathrm{e}}$ & $27.6 \%(\mathrm{~N}=35)$ & $20.7 \%(N=29)$ & 1.71 , n.s. \\
\hline Urine Purine $e^{e}$ & $1.6 \%(\mathrm{~N}=2)$ & $0.7 \%(\mathrm{~N}=1)$ & 0.44, n.s. \\
\hline
\end{tabular}

${ }^{a} A A N / C N S$ recommended in all cases per 2003 Guidelines.

${ }^{b}$ AAN/CNS recommended in all cases per 2011 Evidence Report.

${ }^{c}$ AAN/CNS recommended in select cases per 2003 Guidelines.

${ }^{\mathrm{A} A \mathrm{AP}}$ recommends in all cases.

${ }^{\text {eAAP }}$ recommends in select cases.

${ }^{f} A A P$ recommends carnitine.

n.s. $=$ non-significant $p$-value.

Table 4: Percent of Sub-specialists Ordering Non-recommended Tests for the Routine Etiologic Evaluation of Intellectual Disability

\begin{tabular}{|c|c|c|c|}
\hline Non-Recommended Test & CN (N=127) & DBP (N=140) & Chi Square, $p$-value \\
\hline \hline EEG & $7.1 \%(N=9)$ & $0.7 \%(N=1)$ & $7.50, p=0.006$ \\
\hline CT Scan & $11.8 \%(N=15)$ & $3.6 \%(N=5)$ & $0.52, p=0.01$ \\
\hline Chromosome 15 duplication & $3.1 \%(N=4)$ & $3.6 \%(N=5)$ & $3.63, p=0.06$ \\
\hline Lead Screening & $27.6 \%(N=35)$ & $38.6 \%(N=54)$ & 0.06 \\
\hline
\end{tabular}

n.s. = non-significant p-value. 
to order a DNA test for Fragile $X\left(X^{2}=7.67, p=0.006\right)$. No significant between-group differences were noted for CMA, test for chromosome 15 duplication, or subtelomeric FISH (Tables 3 and 4). With respect to common genetic testing other than DNA for Fragile $\mathrm{X}$, no overall differences between disciplines were noted: $71.6 \%(91 / 127)$ of $\mathrm{CN}$ and $75.7 \%(106 / 140)$ of DBP ordered a karyotype and/or CMA $\left(x^{2}=0.57\right.$, n.s.). A trend was noted for DBP to order a karyotype more frequently than $\mathrm{CN}$, and DBP more commonly ordered a karyotype in conjunction with a CMA than did $\mathrm{CN}$.

\section{EEG and Neuroimaging}

Overall, very few clinicians indicated that they would order an EEG when evaluating a child with ID and no history of seizures or regression. $\mathrm{CN}$ were significantly more likely than DBP to order an EEG $\left(x^{2}=7.50\right.$, $p=0.006$ ). Likelihood to order an EEG was not significantly influenced by the clinician's practice setting - specifically whether or not they were in private practice. Of the $\mathrm{CN}$ who indicated they would order an EEG, $67 \%$ were in private practice, compared to $33 \%$ of the overall $\mathrm{CN}$ sample $\left(X^{2}=2.00\right.$, n.s.).

Of all respondents, $31.1 \%$ indicated they would order one or more neuroimaging studies - with $23.6 \%$ ordering only an MRI (63/267), $7.1 \%$ ordering a CT and an MRI (19/267), and 1 respondent (a DBP) ordering a CT but no MRI. CN were significantly more likely than DBP to order an MRI $\left(X^{2}=42.55, p<0.0001\right)$ and/or CT scan $\left(x^{2}=6.52, p=0.01\right)$ (See Tables 3 and 4$)$. Practice setting did not influence the likelihood of ordering a brain MRI. Specifically, there was no difference in the percentage of $\mathrm{CN}$ who were hospital-based when comparing $\mathrm{CN}$ who would order an MRI (58.7\%) with the entire $\mathrm{CN}$ sample (55.5\%).

\section{Metabolic Testing}

Overall, most respondents indicated they would order no metabolic tests for this hypothetical case. A lead assay was the most commonly ordered metabolic test -- but only one-third of clinicians $(33.3 \%)$ would routinely order this assay. Relatively few clinicians indicated they would order other, more specialized metabolic tests. Some differences between subspecialties were noted. $\mathrm{CN}$ were significantly more likely than DBP to routinely order the following select metabolic tests: serum carnitine $\left(x^{2}=5.16, p=0.02\right)$, urine creatine $\left(x^{2}=8.48, p=0.004\right)$, and urine ammonia $\left(X^{2}=4.36, p=0.04\right)$ (Table 3$)$. Although not significant, DBP tended to order a lead level more frequently than
$\mathrm{CN}$. No significant between-group differences were noted regarding urine organic acids, serum amino acids, or urine purines.

\section{Compliance with Published Guidelines}

For both $\mathrm{CN}$ and $\mathrm{DBP}$, full compliance with published clinical guidelines was very low. None of the $127 \mathrm{CN}$ were completely compliant with the 2003 AAN/CNS guidelines which recommended karyotype and did not recommend CMA (Figure 1a). About half of $\mathrm{CN}$ were considered non-compliant after the first recommendation - ordering a brain MRI. There was also a 3.5-fold decrease in compliance when $\mathrm{CN}$ were asked about ordering non-recommended testing for subtelomeric rearrangements. When compliance was re-assessed using the updated recommendation for genetic testing contained in the 2011 evidence report (CMA and neither karyotype nor test for subtelomeric rearrangements), complete compliance was $7.1 \%$ (Figure 1b). Nearly $40 \%$ of $\mathrm{CN}$ were non-compliant based on their failure to order the first-line CMA. CN also notably dropped off in compliance by not ordering the recommended brain MRI (1.5-fold decrease) and ordering other non-recommended tests (2-fold decrease).

When compliance for DBP was evaluated relative to the AAP's 2014 guidelines, only $11.4 \%$ of DBP were completely compliant (Figure 2). Similar to CN's compliance when assessed against the AAN/CNS' most recent recommendations, DBP's compliance dropped off by more than $30 \%$ due to not routinely ordering a CMA. There was also a 2-fold decrease in compliance because of DBP who routinely order the outdated karyotype. Another 2-fold decrease in compliance resulted from DBP who routinely order additional non-recommended tests.

Between-group differences in complete compliance were noted. When comparing $\mathrm{CN}$ compliance with 2003 AAN/CNS guidelines (0\%) and DBP compliance with 2014 AAP guidelines (11.4\%), CN were significantly less compliant $\left(x^{2}=15.44, p=0.00009\right)$. However, no between-group differences were noted when CN compliance with the 2011 evidence report was compared with DBP compliance with 2014 AAP guidelines $\left(x^{2}=1.48\right.$, n.s. $)$.

For the sample overall, compliance with guidelines did not differ between physicians in practice for 15 years or less when compared to those in practice for 16 years or more $\left(x^{2}=1.13, p=0.29\right)$. Similarly, there were 


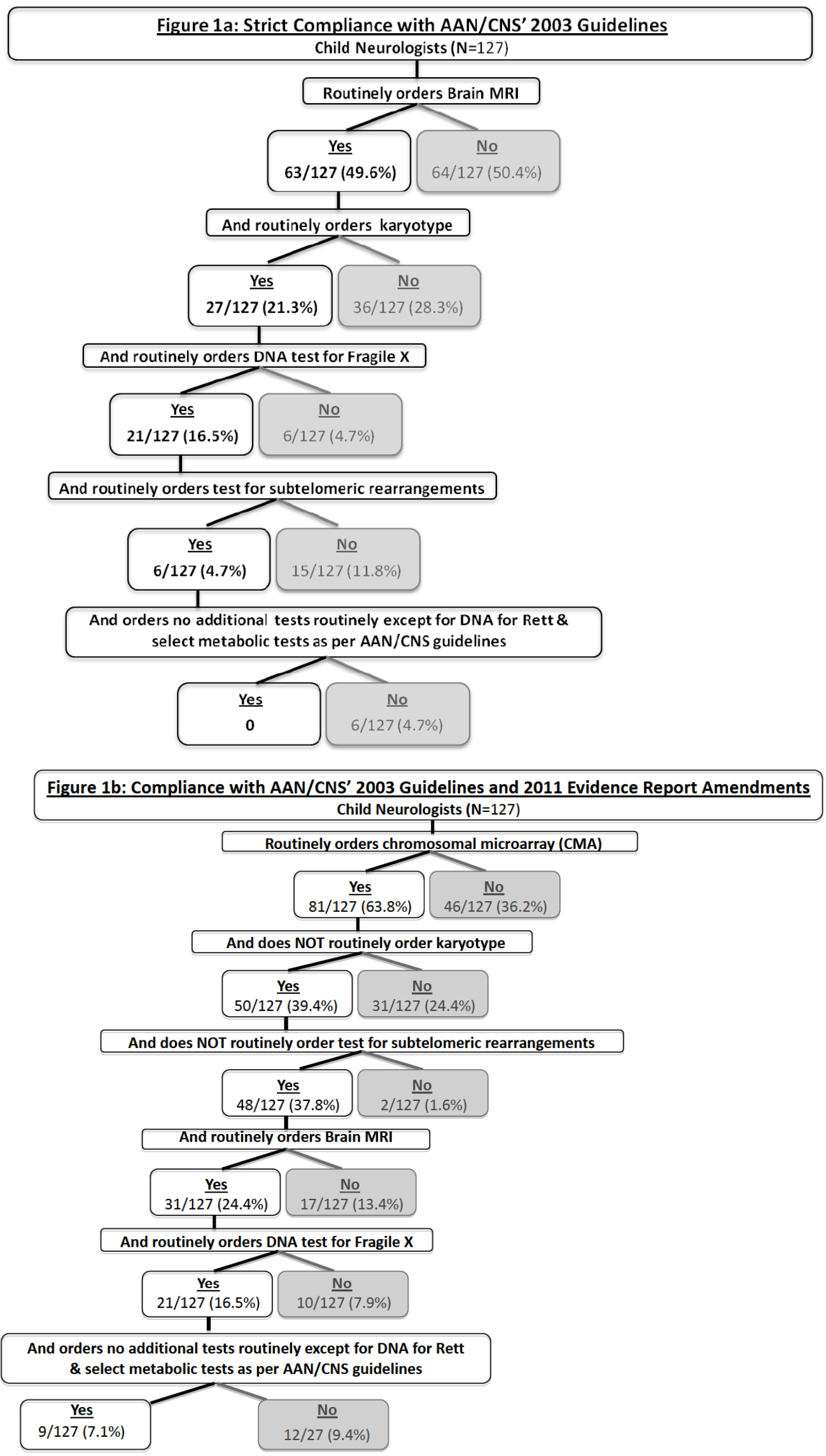

Figure 1: 


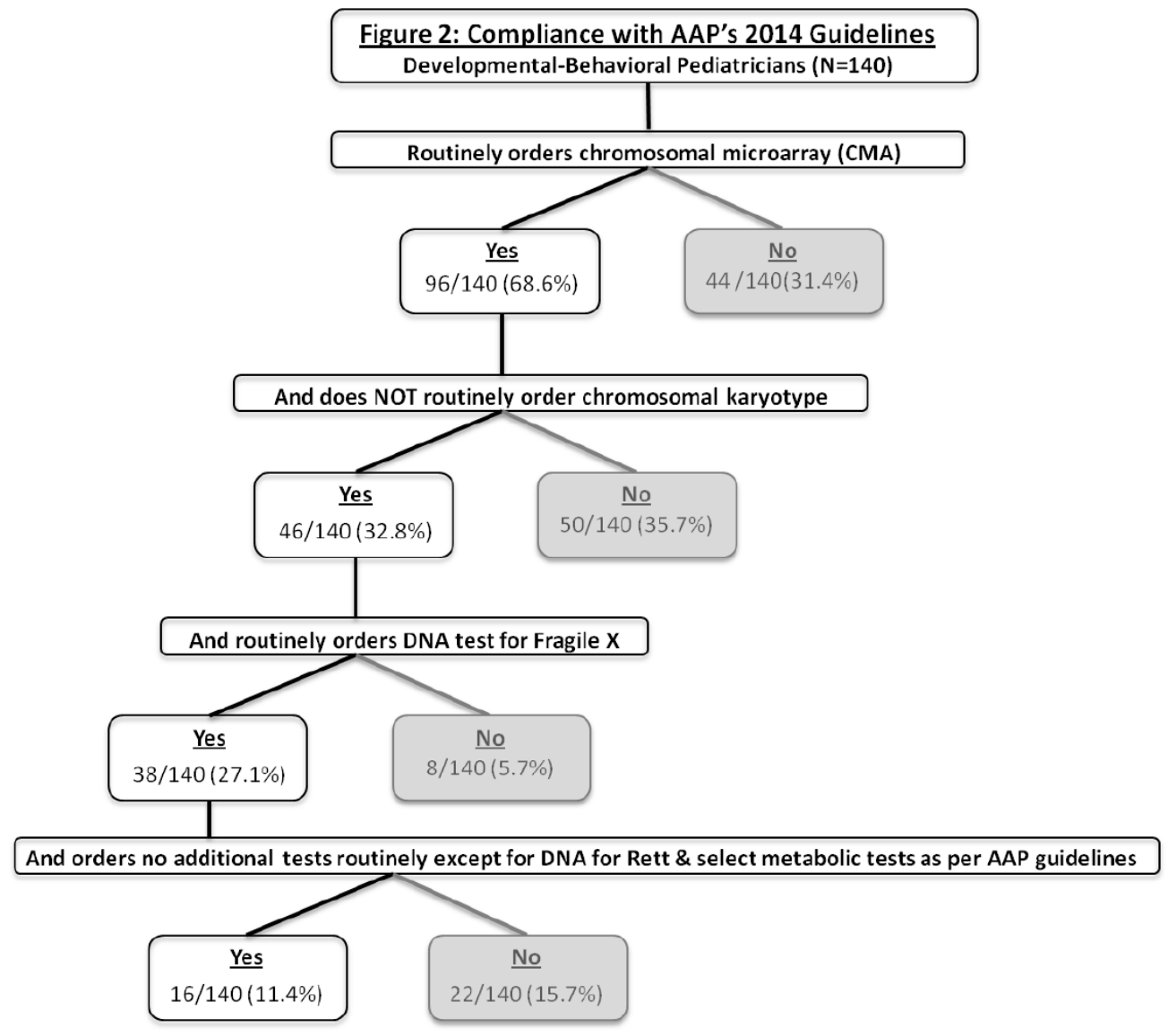

Figure 2:

no differences in compliance with guidelines based on clinician gender.

Compliance across discipline-specific guidelines was also assessed. Though $\mathrm{CN}$ complied with updated guidelines from the AAN/CNS more than with guidelines from the AAP $(7.1 \%$ vs. $5.5 \%)$, these differences were not significant $\left(X^{2}=0.27\right.$, n.s. $)$. A similar same-discipline bias in following guidelines was noted for DBP, who followed the AAP guidelines modestly more than the latest AAN/CNS guidelines $(11.4 \%$ vs. $5.7 \%)$, which was a trend $\left(x^{2}=2.92, p=0.09\right)$.

\section{DISCUSSION}

Laboratory evaluation of children with intellectual disability or global developmental delay is important for a multitude of clinical reasons. Identification of a specific etiology can have meaningful and sometimes profound implications for the child and his family. In some cases, parental guilt may be alleviated, family planning may be influenced, and medical surveillance and/or anticipatory guidance can be individualized. $\mathrm{CN}$ and DBP are the two pediatric specialists most commonly consulted for laboratory evaluation of CWID, and their respective medical societies have issued assessment guidelines or practice parameters to assist in this process. These guidelines not only suggest which tests are indicated in the routine evaluation of a young child with ID, but they also identify tests that should only be ordered in select clinical circumstances and not routinely.

The primary objective of this study was to identify which laboratory tests pediatric specialists routinely recommend for the evaluation of a child with ID and an otherwise unremarkable neurological history and exam. For each of the different major types of tests, discussion follows about our results and their clinical significance and implications.

\section{Genetic Testing}

Until recently, guidelines for genetic testing of children with ID have focused on chromosomal (Gbanded) karyotype as well as testing for Fragile $X$ syndrome [8]. According to the newest genetic guidelines [21], chromosomal microarray analysis (CMA), otherwise known as comparative genomic hybridization $(\mathrm{CGH})$, is the most accurate genetic test 
for identifying ID-associated chromosomal abnormalities. In at least $10 \%$ of patients with severe developmental problems (i.e., intellectual disability, congenital abnormalities, developmental delay, etc.) CMA detects clinically significant copy-number variants with 100 times greater resolution than chromosomal karyotyping [22]. Given its higher yield, it is recommended that CMA, not G-banded karyotypes, now be used as a first-line genetic test for chromosomal abnormalities [14, 21, 23]. When the AAP formally updated its guidelines for ID evaluation in 2014, it likewise included an explicit recommendation for CMA as a first-line test, replacing the standard karyotype and subtelomeric fluorescent in situ hybridization tests [7]. Given the sensitivity of CMA for identifying individuals who are phenotypically normal or have non-specific genetic abnormalities, it is important that all children with otherwise unexplained ID have a CMA performed. Although the majority of subspecialists routinely ordered a CMA as part of their evaluation of CWID, a very large percentage of $\mathrm{CN}$ and DBP did not recommend a CMA in this hypothetical case. To the extent that a third of respondents indicated they would not order a CMA, this suggests that genetic abnormalities could go undiagnosed in a large number of CWID referred to $\mathrm{CN}$ and DBP for medical evaluation - if this sample is representative of the respective subspecialties.

The same was true for DNA testing for Fragile $X$ syndrome -- a genetic disorder found in $\sim 3 \%$ of male and $2 \%$ of female ID cases and the most common inherited etiology of ID [7, 8]. Although this test has been recommended by both the AAN/CNS and AAP for more than 10 years, it is concerning that more than one-third of responding physicians indicated that they would not routinely order this genetic test for CWID. Since both professional societies have endorsed this test for such a long interval, it is unclear why fewer $\mathrm{CN}$ order this test than DBP.

Even though both professional organizations endorse genetic testing as part of the routine evaluation of a young child with ID, approximately onefifth of responding pediatric specialists (CN and DBP) indicated that they would not order any genetic test whatsoever. This may be because these clinicians are deferring the medical work-up to clinical geneticists. When asked separately how often they refer a "child with moderately severe autism or intellectual disability," $88.2 \%$ of the 59 respondents who indicated they would not order any genetic tests indicated that they "sometimes" or "often" refer to "a geneticist for genetic or metabolic testing." Interestingly, this referral rate is similar to the rate at which respondents who order one or more recommended genetic test refer to a geneticist $\left(86.8 \%, x^{2}=0.07\right.$, n.s. $)$.

Just as a third of physicians are erring in not ordering a CMA, a similar percentage of physicians are erring on doing too many genetic tests; $30 \%(81 / 267)$ of the respondents reported they would do a CMA in addition to a karyotype. Neither the AAP nor the AAN/CNS guidelines recommend both of these genetic tests as part of the initial evaluation of CWID. Excessive genetic testing was especially notable for the DBP; more than half of DBP who ordered a CMA also ordered a karyotype.

\section{EEG and Neuroimaging}

The AAN/CNS and AAP guidelines each suggest that, when evaluating ID, an EEG should only be ordered upon presentation of specific symptoms, such as clinical seizures or suspicion of subclinical seizures $[7,8]$. Since $19 \%$ of children with regression display abnormal epileptiform EEGs, EEGs can identify or rule out other disorders that involve seizures and are often mistaken for ID, such as Landau-Kleffner syndrome [24]. Although ordering an EEG would be appropriate if a child with suspected ID has a history of seizures and/or regression, EEGs are not routinely advised for the evaluation of children with ID that lack frank neuropathology $[25,26]$, such as the hypothetical child presented in our questionnaire. In our study, the overwhelming majority of $\mathrm{CN}$ and DBP indicated they would not order an EEG when evaluating a child with $I D$ and no seizures or regression. Though $C N$ were more likely to order an EEG than DBP, the percentages are extremely low. Among $\mathrm{CN}$ who indicated they would order an EEG, the percentage in private practice was twice that of the $\mathrm{CN}$ sample; however, this was not statistically significant - likely because of the very low number of $\mathrm{CN}$ involved who would do an EEG $(n=9)$.

Routine neuroimaging is not recommended by the AAP. By contrast, AAN/CNS guidelines do recommend a brain MRI for unexplained cases of ID, especially when initial genetic tests are negative $[8,9]$. The data from many of the studies that the AAN/CNS cite in support of a brain MRI as a first-line neuroimaging test have small sample sizes that are primarily composed of children with neurologic symptoms [7, 8]. Further, even though $\sim 30 \%$ of children with ID have abnormal findings on a brain MRI, these findings only lead to an etiologic or syndromic diagnosis in a small fraction of such cases [7]. 
Given these discordant recommendations from the two academies, we anticipated that $\mathrm{CN}$ would order neuroimaging tests much more frequently than would DBP. Whereas DBP were more likely to order genetic tests that are not indicated, $\mathrm{CN}$ were, overall, more likely to order "neurologic tests" - EEGs and neuroimaging. Compared to DBP, CN order EEGs, CT scans, and MRIs ten times, three times, and four times more frequently (Tables 3 and $\mathbf{4}$ ).

The fact that only $50 \%$ of $\mathrm{CN}$ indicated they would routinely order a brain MRI may reflect a limitation of the structure of the questionnaire. Since physicians were asked to check off each of the tests they would order, the questionnaire did not provide any context or conditionality for ordering any single test. Since the newest AAN/CNS recommendations stipulate that a brain MRI should only be done if the initial genetic work-up is negative, it is possible some $\mathrm{CN}$ indicated they would not order an MRI since they could not assume a negative genetic work-up. In terms of DBP, $13 \%$ responded that they would order a brain MRI. The clinical rationale for this is unclear since AAP guidelines do not recommend any routine neuroimaging. Although the index child in this case did not have macrocephaly, there was a difference in relative size between the child's head circumference $\left(90^{\text {th }}\right.$ percentile) and height $\left(50^{\text {th }}\right.$ percentile). Some DBP may have thus considered this child to have macrocephaly in a relative if not absolute sense. In a previous study, it was shown that $\mathrm{CN}$ routinely order neuroimaging in patients with autism and macrocephaly to determine if hydrocephalus is present [25]. It is possible that non-clinical factors such as medico-legal considerations may also have influenced some physicians' approach to an etiologic evaluation.

\section{Metabolic Testing}

Since metabolic disorders are the cause of ID in up to $5 \%$ of all cases [9], these tests can be useful for clinicians when there is no clear explanation for a patient's ID. CN tend to order metabolic tests more frequently than DBP, especially tests for carnitine, creatine, and ammonia levels, which can potentially be attributed to the AAN/CNS's recent evidence report on the etiologic yield of these metabolic tests. Both the AAN/CNS and AAP recommend select metabolic testing on a case-specific basis, which include tests for organic acids, amino acids, purine, carnitine, creatine, and ammonia $[7,8]$. Our data suggest that the overwhelming majority of $\mathrm{CN}$ and $\mathrm{DBP}$ are following guidelines and not routinely ordering these tests. We suspect that the step-wise evaluation algorithms presented in both guidelines may account for the low rate at which $\mathrm{CN}$ and DBP ordered these metabolic tests, which are recommended only later in the evaluation process. Some respondents may have assumed that the straightforward case presented in the questionnaire would require only one or two tests to determine the etiology of ID.

\section{Compliance with Guidelines}

Compliance with guidelines was surprisingly low. For CN, we had expected compliance with the 2003 AAN/CNS guidelines to be very low since we presumed most $\mathrm{CN}$ would be ordering a CMA and not a karyotype. This was not the case; a third of $\mathrm{CN}$ reported ordering karyotypes routinely and a third also reported not routinely ordering a CMA. CN compliance with updated recommendations based on the 2011 evidence report was also extremely low. Even when $\mathrm{CN}$ were appropriately ordering a CMA, many $\mathrm{CN}$ were routinely ordering a karyotype in addition or failing to order a brain MRI or DNA for Fragile X.

Guideline compliance was equally low for DBP. Although a higher proportion of DBP ordered CMA, more than half of these physicians indicated that they would routinely order a karyotype in addition to the CMA. In contrast to $\mathrm{CN}$, very few DBP indicated they would routinely order a brain MRI. This is consistent with AAP guidelines, which do not recommend routine neuroimaging. The greatest point of divergence between the AAN/CNS evidence report and the AAP guidelines surrounds the role of neuroimaging. Since both organizations are putting forth evidence-based guidelines, it would be ideal if the two organizations could agree on a common protocol for neuroimaging and other diagnostic approaches.

\section{STUDY LIMITATIONS AND FUTURE DIRECTIONS}

This study represents the first attempt at a systematic evaluation of which laboratory tests that $\mathrm{CN}$ and DBP recommend for the evaluation of ID. Although our findings are striking -- suggesting that $\mathrm{CN}$ and DBP frequently do not order recommended molecular genetic tests and, at the same time, routinely ordering tests that are outdated or otherwise not indicated, our study does have several methodological limitations.

In terms of our sample, a low overall response rate $(16.9 \%)$ may have made our sample less representative of $\mathrm{CN}$ and $\mathrm{DBP}$ nationally - though the 
fact that we received responses from more than 300 physicians out of 1897 physicians approached to participate and had a final sample of 267 actively practicing, board-certified pediatric specialists is not insignificant. Additional information about respondents would have been helpful, including whether they were familiar with discipline-specific guidelines for evaluation of ID and how many children in a week or month they typically see for evaluation of ID. It would have been interesting to look at the relationships between clinicians' familiarity with published guidelines, the volume of patients they see with ID, and their clinical approach to the hypothetical case presented.

Second, as discussed, the index case for this study was a boy with a head circumference of $90 \%$ and height of $50 \%$; although this does not meet criteria for macrocephaly, there is a considerable percentile discrepancy between height and head circumference and this may have influenced some clinicians' responses regarding neuroimaging. In hindsight, it would have been preferable to identify the boy's head circumference as being $75 \%$ or less to avoid clinical ambiguity.

A third consideration relates to our assessment of guideline compliance relative to the stated purpose and timing of these documents. Whereas the AAN disseminated "guidelines" in 2003, subsequent publications related to laboratory testing of ID were not disseminated as "guidelines" per se; instead, in 2011, the AAN/CNS published an "evidence report" and then, two years later, they disseminated a "template coverage policy" - both of which contained recommendations for lab testing. For DBP, we evaluated compliance with "practice parameters" published by the AAP in 2014 - one year after our data was collected. Although we assessed DBP compliance with guidelines that had not yet been published, the AAP's 2014 practice parameters, in essence, codified existing expert consensus at the time that our data was collected. It would be interesting to re-survey this cohort and see if there is greater compliance with guidelines.

Perhaps the greatest limitation of this study was the structure of the questionnaire. Physicians were provided with a broad list of diagnostic tests and asked to check off which test(s) they routinely order. Since the AAN/CNS guidelines are stepped recommendations and we did not provide any if/then conditional information (e.g., if genetic testing was negative, then what other tests would you order), the structure of our questionnaire makes it difficult to be certain what was the basis for responses from $\mathrm{CN}$. If one were to replicate this study, it would be more prudent to do it with a web-based survey that incorporates "skip-logic" - a survey feature that allows a respondent's answer to one question to influence or determine what follow-up questions are asked. This format would also remedy another shortcoming of our questionnaire. Since respondents only had to make a pencil mark in the affirmative, we had to assume that unmarked lab tests would not be ordered; it is conceivable that, in some cases, respondents skipped one or more items. Although we could not adjust our analyses for the possibility that one or more items were skipped and may not truly represent "errors of omission", the study still provides clinical valuable information since it has revealed that many clinicians who are presumed to have expertise with evaluation of children with ID are routinely making "errors of commission" -- ordering tests that are not indicated. It is for this reason that we are confident that our findings are of value and should not only prompt further assessment but should also lead to greater educational efforts.

\section{CONCLUSIONS}

A focused and systematic approach to the laboratory evaluation of a child with ID is important. Although primary care pediatricians are generally able to order the routinely recommended genetic tests (CMA and DNA for Fragile $X$ ), general pediatricians often refer children with global developmental delay or intellectual disability to $\mathrm{CN}$ and/or DBP for assessment and diagnostic work-up. Thus, pediatric sub-specialists play a most important role in the medical evaluation of children with ID.

The AAN/CNS and the AAP have created guidelines for pediatricians and child neurologists to follow when evaluating CWID. Our data resoundingly demonstrates that many pediatric specialists -- when evaluating children with ID -- commit errors of commission by too frequently ordering outdated tests (e.g., karyotypes) and errors of omission by not routinely ordering recommended tests (e.g., chromosomal microarray and DNA test for Fragile X). Although we cannot determine if this reflects a 'knowledge deficit' or a 'practice gap' on the part of practitioners, it is clear that there needs to be greater emphasis on physician education.

As clinical guidelines continue to evolve with diagnostic advances, pediatric specialists must remain 
up-to-date on these changes. Though the AAN/CNS and the AAP have taken different approaches to keeping physicians informed about appropriate medical tests, the very wide gap in clinician compliance with these guidelines suggests the current approach is not working. Medical specialty societies must re-evaluate their current mechanisms for promulgating current and future clinical guidelines. With time, laboratory assessment algorithms for medical evaluation of ID may be incorporated within electronic health records automatically providing clinicians with the latest, evidence-based guidelines about which tests are indicated - routinely or otherwise. Until then, there needs to be a much greater emphasis on educating $\mathrm{CN}$ and DBP about current clinical guidelines; this can likely be accomplished through review articles in academic journals, presentations at local and national professional meetings, and other platforms for continuing medical education.

\section{REFERENCES}

[1] Curry CJ, Stevenson RE, Aughton D, Byrne J, Carey JC, Cassidy $\mathrm{S}$, et al. Evaluation of mental retardation: recommendations of a Consensus Conference: American College of Medical Genetics. Am J Med Genet 1997; 72(4): 468-77.

http://dx.doi.org/10.1002/(SICI)1096-

8628(19971112)72:4<468::AID-AJMG18>3.0.CO;2-P

[2] Shaffer LG, American College of Medical Genetics Professional Practice and Guidelines Committee. American College of Medical Genetics guideline on the cytogenetic evaluation of the individual with developmental delay or mental retardation. Genet Med 2005; 7(9): 650-4.

http://dx.doi.org/10.1097/01.gim.0000186545.83160.1e

[3] Tirosh E, Jaffe M. Global developmental delay and mental retardation--a pediatric perspective. Dev Disabil Res Rev 2011; 17(2): 85-92.

http://dx.doi.org/10.1002/ddrr.1103

[4] American Association on Intellectual and Developmental Disabilities (AAIDD). Frequently Asked Questions on Intellectual Disability. http://aaidd.org/intellectual-disability/ definition/faqs-on-intellectual-disability\#.VRMpUfzF9WI. Acccessed 12 December 2014.

[5] American Psychiatric Association (APA). DSM-5 Intellectual Disability Fact Sheet. http://www.dsm5.org/documents/ intellectual\%20disability\%20fact\%20sheet.pdf. Accessed on 12 December 2014.

[6] Johnson JG, J. Developmental assessment in clinical child psychology. Pergamon Press: New York 1990; pp. 109-127.

[7] Moeschler JB, Shevell M, Committee on Genetics. Comprehensive evaluation of the child with intellectual disability or global developmental delays. Pediatrics 2014; 134(3): e903-18.

http://dx.doi.org/10.1542/peds.2014-1839

[8] Shevell M, Ashwal S, Donley D, Flint J, Gingold M, Hirtz D, et al. Practice parameter: evaluation of the child with global developmental delay: report of the Quality Standards Subcommittee of the American Academy of Neurology and The Practice Committee of the Child Neurology Society. Neurology 2003; 60(3): 367-80.

http://dx.doi.org/10.1212/01.WNL.0000031431.81555.16
[9]

Michelson DJ, Shevell MI, Sherr EH, Moeschler JB, Gropman AL, Ashwal S. Evidence report: Genetic and metabolic testing on children with global developmental delay: report of the Quality Standards Subcommittee of the American Academy of Neurology and the Practice Committee of the Child Neurology Society. Neurology 2011; 77(17): 1629-35.

http://dx.doi.org/10.1212/WNL.0b013e3182345896

[10] Shevell M. Global developmental delay and mental retardation or intellectual disability: conceptualization, evaluation, and etiology. Pediatr Clin North Am 2008; 55(5): 1071-84, xi.

http://dx.doi.org/10.1016/j.pcl.2008.07.010

[11] Boyle CA, Boulet S, Schieve LA, Cohen RA, Blumberg SJ, Yeargin-Allsopp $\mathrm{M}$, et al. Trends in the prevalence of developmental disabilities in US children, 1997-2008. Pediatrics 2011; 127(6): 1034-42. http://dx.doi.org/10.1542/peds.2010-2989

[12] Mefford HC, Batshaw ML, Hoffman EP. Genomics, intellectual disability, and autism. N Engl J Med 2012; 366(8): 733-43.

http://dx.doi.org/10.1056/NEJMra1114194

[13] Duker AL, Teed LN, Thomas RL, Majkowski ME, Bawle EV The cost and yield of evaluations for developmental delay/mental retardation. Dev Med Child Neurol 2008; 50(10): 798-9.

http://dx.doi.org/10.1111/j.1469-8749.2008.03087.x

[14] Christa W, Habela AH. Genetic testing for intellectual disability: A role in diagnostic evaluation. Contemporary Pediatrics 2013. Accessed on 10 January 2015.http://contemporarypediatrics.modernmedicine.com/con temporary-pediatrics/news/RC/genetic-testing-intellectualdisability-role-diagnostic-evaluation?page=full.

[15] Moeschler JB, Shevell M, American Academy of Pediatrics Committee on Genetics. Clinical genetic evaluation of the child with mental retardation or developmental delays. Pediatrics 2006; 117(6): 2304-16. http://dx.doi.org/10.1542/peds.2006-1006

[16] Srour M, Mazer B, Shevell MI. Analysis of clinical features predicting etiologic yield in the assessment of global developmental delay. Pediatrics 2006; 118(1): 139-45.

http://dx.doi.org/10.1542/peds.2005-2702

[17] Satya-Murti S, Cohen BH, Michelson DJ. Template Coverage Policy: Chromosomal microarray analysis for intellectual disabilities. American Academy of Neurology 2013. https://www.aan.com/uploadedFiles/Website_Library_Assets/ Documents/3.Practice_Management/1.Reimbursement/1.Billi ng_and_Coding/5.Coverage_Policies/13\%20ChromoMicrolnt elDisabil.pdf

[18] Bale JF, Jr., Caplin DA, Bruse JD, Folland D. Practice parameters in child neurology: do pediatricians use them? J Child Neurol 2009; 24(12): 1482-5.

http://dx.doi.org/10.1177/0883073809332766

[19] Trivedi MH, Claassen CA, Grannemann BD, Kashner TM, Carmody TJ, Daly E, et al. Assessing physicians' use of treatment algorithms: Project IMPACTS study design and rationale. Contemp Clin Trials 2007; 28(2): 192-212. http://dx.doi.org/10.1016/j.cct.2006.08.005

[20] Bettinger TL, Crismon ML, Trivedi MH, Grannemann B, Shon $\mathrm{SP}$. Clinicians' adherence to an algorithm for pharmacotherapy of depression in the Texas public mental health sector. Psychiatr Serv 2004; 55(6): 703-5. http://dx.doi.org/10.1176/appi.ps.55.6.703

[21] Kearney HM, Thorland EC, Brown KK, Quintero-Rivera F, South ST, Working Group of the American College of Medical Genetics Laboratory Quality Assurance Committee. American College of Medical Genetics standards and guidelines for interpretation and reporting of postnatal 
constitutional copy number variants. Genet Med 2011; 13(7):

680-5.

http://dx.doi.org/10.1097/GIM.0b013e3182217a3a

[22] Shen Y, Dies KA, Holm IA, Bridgemohan C, Sobeih MM, Caronna EB, et al. Clinical genetic testing for patients with autism spectrum disorders. Pediatrics 2010; 125(4): e727-35. http://dx.doi.org/10.1542/peds.2009-1684

[23] Manning M, Hudgins L, Professional P, Guidelines Committee. Array-based technology and recommendations for utilization in medical genetics practice for detection of chromosomal abnormalities. Genet Med 2010; 12(11): 742-5. http://dx.doi.org/10.1097/GIM.0b013e3181f8baad

[24] Ballaban-Gil K, Tuchman R. Epilepsy and Epileptiform EEG: Association with Autism and Language Disorders. Ment
Retard Dev Disabil Res Rev 2000; 6: 300-8. http://dx.doi.org/10.1002/1098-2779(2000)6:4<300::AIDMRDD9>3.0.CO;2-R

[25] Filipek PA, Accardo PJ, Ashwal S, Baranek GT, Cook EH Jr., Dawson G, et al. Practice parameter: screening and diagnosis of autism: report of the Quality Standards Subcommittee of the American Academy of Neurology and the Child Neurology Society. Neurology 2000; 55(4): 468-79. http://dx.doi.org/10.1212/WNL.55.4.468

[26] Johnson CP, Myers SM, American Academy of Pediatrics Council on Children With Disabilities. Identification and evaluation of children with autism spectrum disorders. Pediatrics 2007; 120(5): 1183-215. http://dx.doi.org/10.1542/peds.2007-2361

Received on 31-03-2015

Accepted on 21-05-2015

Published on 07-08-2015

DOI: http://dx.doi.org/10.6000/2292-2598.2015.03.02.9

(c) 2015 Tchaconas and Adesman; Licensee Lifescience Global.

This is an open access article licensed under the terms of the Creative Commons Attribution Non-Commercial License (http://creativecommons.org/licenses/by-nc/3.0/) which permits unrestricted, non-commercial use, distribution and reproduction in any medium, provided the work is properly cited. 